\title{
Interventional oncology: the future
}

\author{
Fredric A. Hoffer
}

Received: 8 December 2010 /Revised: 4 January 2011 / Accepted: 13 January 2011

(C) Springer-Verlag 2011

\begin{abstract}
Interventional radiology techniques to treat oncological disease have already shown value in adults. The adoption and development of interventional oncology (IO) in children have been more limited and challenging. This relates to the approval process for new devices and agents, oncology group protocol limitations and the inherent hesitation of trying new treatments in children. This paper will discuss how new procedures are developed and approved, and the new therapies that will become available to better treat pediatric malignancies. Bringing the benefits of IO to children will require initiative on the part of pediatric diagnostic and interventional radiologists as well as the cooperation of our clinical colleagues.
\end{abstract}

Keywords Interventional oncology Cancer C Children

\section{The challenge}

Unfortunately, many pediatric interventional radiologists are frequently limited in introducing novel oncological interventions by their institutions' pediatric oncologists and surgeons. The future of pediatric interventional oncology (IO) depends on the initiative of pediatric radiologists, pediatric interventional radiologists, pediatric surgeons and pediatric radiation oncologists. We must either lead the adult experience as our pediatric oncologists now do, or follow the adult experience as commonly occurs in radiology.

Disclaimer Dr. Hoffer has no financial interests, investigational or off-label uses to disclose.

\section{F. A. Hoffer $(\square)$}

Department of Radiology, University of Washington,

3904 NE Belvoir Place,

Seattle, WA 98105, USA

e-mail: fhoffer@gmail.com

\section{The process}

Nearly all therapeutic pediatric oncology is performed under prospective clinical trials and protocols associated with large cooperative oncology groups (often funded by individuals, special interest associations and government grants) after scrutiny by scientific committees and institutional review board (IRB) approval. Protocol development is expected to first involve animal studies, then adult studies, before progressing to children. Trials are also phased for the protection of participants. New agents are first examined for toxicity in a Phase I trial, examined for efficacy (e.g. tumor response) in a Phase II trial, and finally examined to determine patient outcome in a Phase III trial. However, in clinical trials, new treatments are given to individual patients in the opposite order. Treatments with proven safety and efficacy are used in a Phase III trial for initial treatment. If the patient has a poor response, undue toxicity or recurrence, they may be switched to a Phase II trial treatment that is known to be safe but not proven to be efficacious. If the patient fails that treatment, they may opt for a Phase I trial knowing that there is no known beneficial effect and that the new treatment may not help.

Unlike much of surgery or radiation oncology, nearly all of IO involves new agents or delivery devices. Although many products are not approved for pediatric use, if specific adult indications are approved by the Federal Drug Administration, FDA approval for similar specific pediatric use may not be required. If the FDA has not approved the drug or device for a patient's specific need, the drug or device may be used off-label according to best clinical management. Using a drug or device in a prospective research trial may require an application to the FDA for an investigational new drug (IND) or investigational device exemption (IDE), respectively. In questionable cases, the 
local IRB may ask the investigator to consult with the FDA to determine if an IND or IDE is necessary.

Because children may not understand research or the nature of their condition and proposed treatment and are dependent on caretakers, they are a vulnerable population and, therefore, receive extra protection under national and international guidelines for human investigation. Pediatric IRBs are charged with determining that the potential benefit of the research to the child outweighs the risk. Assent from the child, in addition to consent from the parent or guardian, is required. Although cooperative cancer research groups such as the Children's Oncology Group (COG) have overcome these obstacles, they do not have resources available to fund pediatric IO trials.

Children are an underserved population when it comes to some types of research. The NIH has specified [1] that children cannot be excluded from NIH-funded studies unless the research topic is irrelevant to children, knowledge sought is already available in children or will be obtained from another ongoing study, a separate age-specific study is warranted and preferable, or insufficient data are available in adults to determine potential risks in children. The American College of Radiology Imaging Network (ACRIN) functions as an open oncology trial resource for radiologists and funds IO trials, but they are limited to adults. The reluctance to include children in trials may be due to difficulties with consent, assent and pediatric IRBs. ACRIN has a subcommittee that deals with monitoring underserved populations, but so far pediatric IO is not being considered.

Other obstacles that limit the future of pediatric IO include pediatric oncologists who prefer systemic chemotherapy rather than imaging-guided local or regional treatments. A chemoembolization arm for primary hepatic malignancies had poor accrual in a prior pediatric oncology group trial, and pediatric oncologists have disapproved intra-arterial chemotherapy for osteosarcoma (personal observation). One must also understand that pediatric oncologists' standard for treatment is protocol driven; they get their hands slapped if they refer their patients for non-protocol treatment.

The newly created Society for Pediatric Interventional Radiology (SPIR, http://spirweb.org) has discussed the need for IO for pediatric patients. Thus it is clear, progress in pediatric IO will involve tedious research protocol development. Who will take the initiative?

\section{Adult IO leads pediatric IO}

Pediatric investigators and practitioners often follow the lead of their adult counterparts. As adult interventional radiologists are losing endovascular work to surgeons, they are turning more to IO. Interventional, cross-sectional, musculoskeletal, breast, GI and GU radiologists are all performing interventional oncology in adults. The recognition of the practice of IO has allowed non-endovascular radiologists to join the Society of Interventional Radiology (www.sirweb.org). Adult radiologists have influenced adult oncologists and surgeons, who in turn will introduce pediatric oncologists and surgeons to new oncological interventions.

Perhaps even more important than the experience and teaching coming from our adult counterparts is their prolific research. There were more IO articles published from the United States from 1996-2008 than from any other country. However, there now more IO clinical research protocols in China and Japan than any other country [2], perhaps due in part to the prevalence of hepatoma. China, in particular, is becoming a powerhouse for drug and device development and was featured at the 2010 annual Radiological Society of North America meeting because of its sophisticated volume of scientific work. If this trend continues, the background work on new therapies may well be performed outside of the United States and Europe. This, in turn, might facilitate FDA approval and allow earlier adoption of these treatments in America and Europe in pediatric clinical trials.

In considering specific IO therapies, let us examine the future and work back to the present.

\section{Theranostic agents and nanoparticles}

Agents that combine therapeutic and diagnostic applications are referred to as theranostic agents. Nanoparticles for anticancer drug delivery may be a technique for directed cancer therapy in the future, and nanomedicine offers a lessinvasive alternative to locoregional treatment. Nanoscale carriers are being tailored to accumulate in tumor cells and tissues by using ligands that recognize tumor-associated antigens [3]. Nanobialys (named after bialystoker, the Yiddish name for a Polish roll with a central depression filled with diced spices) are capable of incorporating chemotherapeutic compounds with greater than $98 \%$ efficiency. When given systemically, $80 \%$ of the drug may be selectively retained by the tumor cell or tissue. If labeled with manganese (Mn [III]), these agents can be imaged by magnetic resonance imaging as it reaches its tumor target [4]. Theranostic agents may involve the diagnostic pediatric radiologist in the future as the evaluator of IO agent delivery.

\section{Irreversible electroporation}

Irreversible electroporation (IRE) is a promising IO technique where two to six probes are placed percutaneously into a tumor, which is then shocked by high-voltage microsecond electrical pulses. This technique causes tumor 
cell apoptosis (progression to irreversible cell death) by having the electrical pulse cause permanent pores in the tumor cell membrane [5]. Reversible electroporation was first described in 1984 [6] and has been used for many purposes, including transfecting viruses into cells for gene therapy [7]. When IRE was recently demonstrated on a porcine liver, the target liver tissue underwent apoptosis, but supporting structures within the ablation zone, such as blood vessels and bile ducts, were spared [8]. The first Phase I study was of renal cell carcinoma treated with IRE immediately before resection. The technique was found to be safe with no major complications [9]. The advantages of this nonthermal ablation technique in pediatric tumor ablation compared to radiofrequency ablation include no grounding pads necessary thus avoiding possible skin burns [10]; no heating occurs avoiding hyperthermia during ablation of large tumors in small children [11]; the procedure is quick avoiding long anesthesia times, and the ablation zone is immediately delineated as a hypoechoic area by ultrasound [8]. A single bipolar probe (NanoKnife, www.Angiodynamics.com, Angiodynamics, Queensbury, N.Y.) is being developed for clinical use.

\section{Radioembolization}

Embolization with radioactive material (radioembolization) of hepatic malignancies has been an alternative to radiofrequency ablation and chemoembolization as an IO technique for adult patients. The advantage of radioembolization is that it is administered via the hepatic artery, which preferentially feeds hepatic tumors rather than hepatic parenchyma. Radioembolization has advantages in patients who do not have a chemosensitive tumor or have reached toxicity limits from prior chemotherapy. More recently, radioembolization with yttrium-90 (Y90) microspheres has been proven to have less toxicity, longer time to disease progression and similar survival for patients with advance hepatocellular carcinoma compared with chemoembolization [12]. Potential complications of Y90 radioembolization include pulmonary irradiation and gastric perforation. Excessive pulmonary or systemic irradiation is avoided by selecting only those patients with small hepatic artery to pulmonary artery shunts as determined by preembolization hepatic artery injection of $99 \mathrm{~m}$-Technitium macroaggregated albumin. Pre-procedure coil embolization of gastrointestinal collaterals prevents gastrointestinal irradiation. Radioembolization combined with systemic chemotherapy has also improved response and survival for adult patients with unresectable colorectal liver metastases [13]. It seems clear that its use will be increasing in the future. At least one pediatric patient has been treated with Y90 (Dr. Manraj Heran, personal communication).

\section{Chemoembolization}

Transcatheter arterial chemoembolization (TACE) has become an important IO tool with more than 300 papers published within the last year. The advantage of combined chemotherapy and embolization is that the arterial administration concentrates the chemotherapy and the embolization increases the dwell time at the target with less systemic effect than intravenous or oral chemotherapy. TACE has been widely used for hepatic colon cancer metastases [14] and hepatocellular carcinoma where the embolic material may be ethiodol, particles, or chemotherapy-eluding beads [15]. TACE has decreased hepatic-prevented progression facilitating orthotopic liver transplantation (OLT) [16]. Presurgical TACE for pediatric hepatoblastoma had no significant chemotoxicity, but decreased tumor volumes allowed complete tumor removal in 10 of 13 children, including one who underwent OLT [17].

TACE has also been used for lung tumors that have a dual supply from the pulmonary and bronchial arteries [18]. TACE has been used in osseous tumors [19] where there is no dual blood supply but where targeted therapy helps maintain organ function. TACE of Wilms tumor has demonstrated safety, prolonged time to tumor progression and increased survival compared to surgery alone [20].

TACE has also been used in combination with systemic chemotherapy for colon metastases [21], and in combination with radiofrequency ablation (RFA) for intermediatesized hepatocellular carcinoma [22]. The combination of TACE and RFA attacks the peripheral perfused tumor and central ischemic portion of the tumor, respectively. TACE prior to RFA also has the advantage of decreasing the heat sink effect of cooling blood flow to allow allow more effective RFA tumor heating.

\section{Intra-arterial chemotherapy}

At present, retinoblastoma is the only pediatric tumor widely accepted for intra-arterial chemotherapy without embolization. Direct ophthalmic artery infusion of melphalan had minimal toxicity in one series of nine cases resulting in the salvage of seven eyes that otherwise would have been enucleated [23]. Intra-arterial chemotherapy combined with osmotic blood-brain barrier disruption has been used to treat embryonal and germ cell tumors of the central nervous system [24]. There are many other papers that describe intraarterial chemotherapy, but except for the eye and brain there is no rationale for avoiding chemoembolization.

Hyperthermic isolated limb perfusion is a technique touted by surgeons where they isolate the major artery and vein of an extremity involved with a malignant or aggressive tumor and then perfuse the extremity with an 
agent, such as tumor necrosis factor or melphalan, in an attempt to necrose the tumor [25]. This allows high-dose regional treatment without systemic effect. More recently, interventional radiologists modified this technique by using an arterial and venous catheter placed from the opposite extremity and cutting off the circulation with a tourniquet proximal to the tips of the catheters (termed isolated limb infusion) [26].

\section{Thermal ablation}

\section{High-intensity focused US (HIFU)}

Magnetic resonance guided high-intensity focused ultrasound (HIFU or MRgFUS) has successfully ablated primary breast cancers in adults [27]. Chinese investigators have used ultrasound-guided HIFU with systemic chemotherapy in a pediatric and young adult trial to successfully treat primary osteosarcoma and Ewing sarcoma of the bone, resulting in complete tumor ablation in 69 of 80 cases $(86 \%)$, and more than $50 \%$ tumor ablation in the remaining 11 patients [28]. The patients were followed for 5 years and the local recurrence rate was $5 / 69(7 \%)$. This technique may be used in the future to prepare patients for limb salvage. Placing a probe on the skin avoids the penetrating percutaneously placed probe into the target used by other thermal ablation techniques, decreasing the potential complications of tumor seeding. HIFU is feasible in any location penetrable by ultrasound including the liver [29], and may be used to ablate brain lesions in the future [30].

\section{Other thermal ablation techniques: pulmonary tumor ablation}

ACRIN is proposing to open an IO protocol for treatment of primary carcinoma of the lung or pulmonary metastases in adults with various thermal ablation techniques that require placing a probe through an intercostal space percutaneously under CT guidance. Microwave has an advantage of completely ablating tumors around large vessels and may be the preferred method for central pulmonary lesions [31]. Cryotherapy has the advantage of being much less painful and may be the preferred technique for ablating pulmonary lesions contiguous with the parietal pleura [32]. Laser also has been used to treat lung metastases in adults [33]. However, RFA is most widely used for ablating lung tumors in adults and children [10] despite complications including diaphragmatic hernia, hemoptosis and air embolus [34]. Despite the complications, pulmonary lesion thermal ablation may become an alternative for pediatric patients with pulmonary metastases, especially after the first thoracotomy.

\section{Future applications and developments in RFA}

Which thermal technique should a pediatric radiologist use? RFA has been FDA approved for general use to ablate soft tissue lesions [35]. Since RFA is the treatment of choice for ablation of osteoid osteoma, many pediatric institutions have acquired RF generators and are beginning to use them as the first choice for thermal ablation of malignant pediatric tumors. RFA has been used to treat Wilms tumor in the kidney [36]. There has been extensive experience of RFA in the adult liver but limited publications in pediatrics [37]. RFA is specifically indicated to ablate unresectable liver lesions and painful bone tumors [38]. Bone tumor ablation has two specific advantages over radiotherapy. Pain relief from RFA is immediate [38] and RFA can target lesions in a more localized fashion than standard radiotherapy.

RFA of malignant lesions of the extremity and the head and neck is limited due to the resistance between the probe and remote grounding pads [10]. Bipolar RFA devices are becoming available that will allow high-energy deposition into tumors in any location without the use of grounding pads [39]. As in the liver, RFA use may be improved by infusion of liposomal doxorubicin chemotherapy killing the periphery of the lesion that currently only suffers reversible heat damage [40, 41]. Cyber-knife, or stereotactic radiotherapy, presently has an advantage of ablating inaccessible lesions in the brain [42] but will compete with other IO procedures in the future.

\section{Chemical ablation}

Chemical ablation with liquid agents has been performed, but can be limited by poor distribution throughout the lesions and poor containment of the agent. Injection of chemical agents, such as ethanol, into localized hepatocellular carcinomas of adult patients has worked well because the surrounding and inciting cirrhosis is tough and contains the alcohol in the softer tumor [43]. In a porcine model, acetic acid and sodium hydroxide were injected separately but simultaneously into a coaxial needle imbedded in the liver [44]. The resultant acidbase interaction caused the tissue to heat up to $60^{\circ} \mathrm{C}$ causing thermochemical ablation and coagulation necrosis and leaving only inert byproducts of salt and water. Acetic acid has also been used to chemically ablate aggressive fibromatosis (desmoid tumor) [45] and ethanol has been used to sclerose vascular malformations [46], but both agents may damage nearby skin and nerves. Given the alternatives, the indications for chemically ablating pediatric malignant lesions do not seem obvious. However, thermochemical ablation may play a role in treating benign lesions such as osteoid osteoma, Langerhans cell histiocytosis or desmoid tumor, in the future. 


\section{The future of assessing response to oncological interventions}

Unlike with surgery, the tumor is not removed with interventional oncology techniques. To assure complete ablation of a microscopic tumor in the periphery of malignant lesions, the ablation zone is made larger than the tumor. It may take 3 to 6 months for the tumor and reaction to decrease to the original size of the tumor prior to ablation [10]. Using size alone is not sufficient to determine response. One has to determine whether the tumor is dead. If the ablation zone is visible by ultrasound (US), color flow US can be used to determine residual disease that warrants additional ablation prior to ending the ablation procedure. However, the most reliable technique and time for determining tumor response by contrast-enhanced US is the day after ablation [47]. After the tumor is ablated, it may also be stiffer compared to normal tissue and US elastography may help determine the completeness of ablation [48]. Contrast agents in CT and MR also help determine complete or incomplete ablation. Incomplete ablation is suggested by nodular enhancement or asymmetric rim enhancement [10]. The ablated area may appear bright on pre- and postcontrast CT and MR images. Therefore, contrast-enhanced CT or MR images are more diagnostic if image subtraction of the noncontrast image is used. However, this subtraction technique can be limited by miss-registration from respiratory motion. Diffusionweighted MRI is also becoming a tool to assess response after IO treatment [49]. FDG-PET is the best current tool to determine completeness of ablation after interventional oncology treatments [50].

\section{Conclusion}

Interventional oncology has already shown value in adults. Limited experience has shown a similar benefit in children. There are challenges of developing appropriate devices and agents suitable for children as well as challenges in convincing pediatric oncologists to incorporate IO therapies into treatments and trials. Pediatric diagnostic and interventional radiologists need to be involved with their clinical colleagues to bring the promises of pediatric IO to fruition.

\section{References}

1. NIH Policy and Guidelines on the Inclusion of Children as Participant in Research involving Human Subjects. Available via http:/grants.nih.gov/grants/guide/notice-files/not98-024.html

2. Chow DS, Itagaki MW (2010) Interventional oncology research in the United States: slowing growth, limited focus, and a low level of funding. Radiology 257:410-417
3. Conti M, Tazzari V, Baccini C et al (2006) Anticancer drug delivery with nanoparticles. In Vivo 20:697-701

4. Pan D, Caruthers SD, Hu G et al (2008) Ligand-directed nanobialys as theranostic agent for drug delivery and manganese-based magnetic resonance imaging of vascular targets. J Am Chem Soc 130:91869187

5. Miller L, Leor J, Rubinsky B (2005) Cancer cells ablation with irreversible electroporation. Technol Cancer Res Treat 4:699-705

6. Sugar IP, Neumann E (1984) Stochastic model for electric fieldinduced membrane pores. Electroporation. Biophys Chemist 19:211-225

7. Knutson JC, Yee D (1987) Electroporation: parameters affecting transfer of DNA into mammalian cells. Anal Biochem 164:44-52

8. Lee EW, Chen C, Prieto VE et al (2010) Advanced hepatic ablation technique for creating complete cell death: irreversible electroporation. Radiology 255:426-433

9. Pech M, Janitzky A, Wendler JJ et al (2011) Irreversible electroporation of renal cell carcinoma: a first-in-man phase I clinical study. Cardiovasc Intervent Radiol 34:132-138

10. Hoffer FA, Daw NC, Xiong X et al (2009) A phase 1/pilot study of radiofrequency ablation for the treatment of recurrent pediatric solid tumors. Cancer 115:1328-1337

11. Hoffer FA, Campos A, Xiong X et al (2007) Core body temperature regulation of pediatric patients during radiofrequency ablation. Pediatr Radiol 37:297-300

12. Salem R, Lewandowski RJ, Kulik L et al (2010) Radioembolization results in longer time-to-progression and reduced toxicity compared with chemoembolization in patients with hepatocellular carcinoma. Gastroenterology Oct 30 [Epub ahead of print]

13. Chua TC, Bester L, Saxena A et al (2010) Radioembolization and systemic chemotherapy improves response and survival for unresectable colorectal liver metastases. J Cancer Res Clin Oncol Sep 22 [Epub ahead of print]

14. Albert M, Kiefer MV, Sun W et al (2011) Chemoembolization of colorectal liver metastases with cisplatin, doxorubicin, mitomycin C, ethiodol, and polyvinyl alcohol. Cancer 117:343-352

15. Grosso M, Vignali C, Quaretti P et al (2008) Transarterial chemoembolization for hepatocellular carcinoma with drug-eluting microspheres: preliminary results from an Italian multicentre study. Cardiovasc Interv Radiol 31:1141-1149

16. Graziadei IW, Sandmueller H, Waldenberger P et al (2003) Chemoembolization followed by liver transplantation for hepatocellular carcinoma impedes tumor progression while on the waiting list and leads to excellent outcome. Liver Transplant 9:557-563

17. Li JP, Chu JP, Yang JY et al (2008) Preoperative transcatheter selective arterial chemoembolization in treatment of unresectable hepatoblastoma in infants and children. Cardiovasc Interv Radiol 31:1117-1123

18. Vogl TJ, Lehnert T, Zangos S et al (2008) Transpulmonary chemoembolization (TPCE) as a treatment for unresectable lung metastases. Eur Radiol 18:2449-2455

19. Chu JP, Chen W, Li JP et al (2007) Clinicopathologic features and results of transcatheter arterial chemoembolization for osteosarcoma. Cardiovasc Interv Radiol 30:201-206

20. Li JP, Chu JP, Oh P et al (2010) Characterizing clinicopathological findings of transarterial chemoembolization for Wilms tumor. J Urol 183:1138-1144

21. You YT, Changchien CR, Huang JS et al (2006) Combining systemic chemotherapy with chemoembolization in the treatment of unresectable hepatic metastases from colorectal cancer. Int J Colorectal Dis 21:33-37

22. Morimoto M, Numata K, Kondou M et al (2010) Midterm outcomes in patients with intermediate-sized hepatocellular carcinoma: a randomized controlled trial for determining the efficacy of radiofrequency ablation combined with transcatheter arterial chemoembolization. Cancer 116:5452-5460 
23. Abramson DH, Dunkel IJ, Brodie SE et al (2008) A phase I/II study of direct intraarterial (ophthalmic artery) chemotherapy with melphalan for intraocular retinoblastoma initial results. Ophthalmology 115:1398-1404

24. Jahnke K, Kraemer DF, Knight KR et al (2008) Intraarterial chemotherapy and osmotic blood-brain barrier disruption for patients with embryonal and germ cell tumors of the central nervous system. Cancer 112:581-588

25. Bonvalot S, Rimareix F, Causeret S et al (2009) Hyperthermic isolated limb perfusion in locally advanced soft tissue sarcoma and progressive desmoid-type fibromatosis with TNF $1 \mathrm{mg}$ and melphalan (T1-M HILP) is safe and efficient. Ann Surg Oncol 16:3350-3357

26. Brady MS, Brown K, Patel A et al (2009) Isolated limb infusion with melphalan and dactinomycin for regional melanoma and softtissue sarcoma of the extremity: final report of a phase II clinical trial. Melanoma Res 19:106-111

27. Furusawa H, Namba K, Nakahara H et al (2007) The evolving non-surgical ablation of breast cancer: MR guided focused ultrasound (MRgFUS). Breast Cancer 14:55-58

28. Chen W, Zhu H, Zhang L et al (2010) Primary bone malignancy: effective treatment with high-intensity focused ultrasound ablation. Radiology 255:967-978

29. Orsi F, Zhang L, Arnone P et al (2010) High-intensity focused ultrasound ablation: effective and safe therapy for solid tumors in difficult locations. AJR 195:W245-W252

30. Jagannathan J, Sanghvi NT, Crum LA et al (2009) High-intensity focused ultrasound surgery of the brain: part 1-A historical perspective with modern applications. Neurosurgery 64:201-211

31. Wolf FJ, Grand DJ, Machan JT et al (2008) Microwave ablation of lung malignancies: effectiveness, CT findings, and safety in 50 patients. Radiology 247:871-879

32. Niu L, Wang J, Zhou L et al (2010) Complications of cryoablation in 644 lung cancer patients and its treatment. Zhongguo Fei Ai Za Zhi 13:832-834

33. Rosenberg C, Puls R, Hegenscheid K et al (2009) Laser ablation of metastatic lesions of the lung: long-term outcome. AJR 192:785-792

34. Burgoyne LL, Pereiras LA, Laningham F et al (2008) Near-fatal acute bronchovenous fistula in a child undergoing radiofrequency ablation of a metastatic lung tumor. Pediatr Anesth 18:131-133

35. Nashida Y, Yamakado K, Kumamoto T et al (2007) Radiofrequency ablation used for the treatment of frequently recurrent rhabdomyosarcoma in the masticator space in a 10-year-old girl. J Pediatr Hematol Oncol 29:640-642

36. Brown SD, Vansonnenberg E, Morrison PR et al (2005) CTguided radiofrequency ablation of pediatric Wilms tumor in a solitary kidney. Pediatr Radiol 35:923-928
37. Ye J, Shu Q, Li M et al (2008) Percutaneous radiofrequency ablation for treatment of hepatoblastoma recurrence. Pediatr Radiol 38:1021-1023

38. Rybak LD, Rosenthal DI, Wittig JC (2009) Chondroblastoma: radiofrequency ablation-alternative to surgical resection in selected cases. Radiology 251:599-604

39. Peng ZW, Liang HH, Chen MS et al (2011) Conformal radiofrequency ablation of hepatocellular carcinoma with a multi-pin bipolar system. J Surg Oncol 103:69-74

40. Head HW, Dodd GD 3rd, Bao A et al (2010) Combination radiofrequency ablation and intravenous radiolabeled liposomal Doxorubicin: imaging and quantification of increased drug delivery to tumors. Radiology 255:405-414

41. Poon RT, Borys N (2009) Lyso-thermosensitive liposomal doxorubicin: a novel approach to enhance efficacy of thermal ablation of liver cancer. Expert Opin Pharmacother 10:333-343

42. Flannery T, Kano H, Martin JJ et al (2009) Boost radiosurgery as a strategy after failure of initial management of pediatric primitive neuroectodermal tumors. J Neurosurg Pediatr 3:205-210

43. Lencioni R, Crocetti L, Cioni D et al (2010) Single-session percutaneous ethanol ablation of early-stage hepatocellular carcinoma with a multipronged injection needle: results of a pilot clinical study. J Vasc Interv Radiol 21:1533-1538

44. Farnam JL, Smith BC, Johnson BR et al (2010) Thermochemical ablation in an ex-vivo porcine liver model using acetic acid and sodium hydroxide: proof of concept. J Vasc Interv Radiol 21:1573-1578

45. Clark TW (2003) Percutaneous chemical ablation of desmoid tumors. J Vasc Interv Radiol 14:629-634

46. Alomari AI, Karian VE, Lord DJ et al (2006) Percutaneous sclerotherapy for lymphatic malformations: a retrospective analysis of patient-evaluated improvement. J Vasc Interv Radiol 17:16391648

47. Zhou P, Kudo M, Minami Y et al (2007) What is the best time to evaluate treatment response after radiofrequency ablation of hepatocellular carcinoma using contrast-enhanced sonography? Oncology 72(Suppl 1):92-97

48. Kolokythas O, Gauthier T, Fernandez AT et al (2008) Ultrasoundbased elastography: a novel approach to assess radio frequency ablation of liver masses performed with expandable ablation probes: a feasibility study. J Ultrasound Med 27:935-946

49. Ouma T, Matsuoka T, Yamamoto A et al (2009) Assessment of early treatment response after CT-guided radiofrequency ablation of unresectable lung tumours by diffusion-weighted MRI: a pilot study. Br J Radiol 82:989-994

50. Wahl RL, Jacene H, Kasamon Y et al (2009) From RECIST to PERCIST: evolving considerations for PET response criteria in solid tumors. J Nucl Med 50(Suppl 1):122S-150S 